\title{
Integrated Approaches for COVID-19 Case Finding and Their Impact on Timeliness for Disease Containment - Changning District, Shanghai Municipality, China, January-July, 2020
}

\author{
Xubin Zheng ${ }^{1,2,8 x}$; Jie Wang ${ }^{3, x}$; Enmao Cai ${ }^{3}$; Yu Jiang ${ }^{1,3}$; Hong Tang ${ }^{3}$; Biao $\mathrm{Xu}^{1,2, *}$
}

\section{Summary}

What is already known on this topic?

The demand for containing the virus and protecting the economy is high on the agenda of policymakers during the coronavirus disease 2019 (COVID-19) pandemic. Modelling studies indicated that highly effective contact tracing and case isolation were enough to contain the spread of COVID-19 at the early stages, but this has not been validated in real world contexts. What is added by this report?

Integrated case finding approaches, including outpatient monitoring, exposed people quarantining, and contact tracing, effectively contained the spread of COVID-19 in a densely populated district in Shanghai Municipality, China. Active case-finding involving quarantine of exposed persons and contact tracing could reduce the time from symptom onset to COVID-19 diagnosis, thus reducing the risk of local transmission.

What are the implications for public health practice?

Active case-finding should be prioritized as an effective approach to minimize the risk of local transmission in future pandemics. Integrated COVID-19 case finding approaches applied in Shanghai may inform public health policy in other regions where strict lockdown is not applicable.

The new wave of coronavirus disease 2019 (COVID-19) has been threatening the global efforts on disease containment since late 2020. This study aimed to delineate the approaches for COVID-19 case finding in its early outbreak stage in a district of Shanghai Municipality, China, and to evaluate the impact of timeliness on COVID-19 containment. The records of case investigations in Changning District were retrospectively reviewed up to July 2020. Casefinding approaches for local transmission included outpatient monitoring, exposed people quarantining, and contact tracing. The paths of detection of all domestic COVID-19 cases in Changning District in 2020 were analyzed. Overall, 22 local COVID-19 cases were identified. From the 6,160 outpatient visits to designated fever clinics, 58 suspected cases were identified and 8 were subsequently confirmed. The number of people travelling to/from epidemic locations and quarantining within the district was 1,570. During the 14-day quarantine, 9 reported symptoms and 8 were confirmed. Of the 27 close contacts living in the district, 8 reported symptoms during quarantine, and 6 were confirmed. The median time from symptom onset to COVID-19 diagnosis was 6 and 2 days for outpatient monitoring and active casefinding $(P=0.003)$, thus reducing the risk of local transmission. The effective containment in Shanghai may inform public health policy in other regions where strict lockdown is not applicable.

As a respiratory infectious disease, the spread of COVID-19 is influenced by human mobility, population density, and age structures (1). The Changning District of Shanghai possesses a population of nearly 690,000 and the population density is as high as around 18,000 per square kilometer. Apart from the high population density, it is also the home to the Hongqiao Integrated Transportation Hub, which handles a large volume of civil aviation, high-speed and interurban railways, long-distance passenger transportation, subways, ground buses, and taxis (2). As the forefront for COVID-19 containment in Shanghai, Changning District faces substantial threats.

Many regions in the world have moved into a period where the new wave of COVID-19 has been threatening the efforts on disease containment since late 2020. The demand for containing the virus and protecting the economy is high on the agenda for policymakers. However, there are few studies yet to help us understand what works, what is necessary, what is sufficient, and what is too much. Modelling studies showed that highly effective contact tracing and 
household quarantine were enough to achieve disease containment (3-4), but these studies need to be validated in real-world contexts.

This study was a secondary data analysis based on the records of COVID-19 case investigations in Changning District, Shanghai, from January 16 up to July 14 2020. Three case-finding approaches were applied simultaneously to rapidly identify COVID-19 cases from the district. The first approach was to strengthen outpatient monitoring. In Changning District, 5 fever clinics in 3 general hospitals, 1 traditional Chinese medicine hospital, and 1 community health center were designated for COVID-19 case finding. The second approach was to quarantine individuals with travel history to Hubei Province (within which Wuhan City is located) over the past 14 days since they were suspected to have possible exposure to COVID-19. The third approach was contact tracing for confirmed COVID-19 cases. The latter two approaches were collectively called active case finding. A 14-day home quarantine was required and recommended for all exposed people and identified close contacts. During quarantine, if an individual reported suspected symptoms, they were promptly transferred to a designated fever clinic for clinical examination. (Figure 1)

Quarantine data were collected from January 25 to February 27, 2020 since no local transmission was reported 2 weeks after the diagnosis of last local case, i.e., February 12, in Changning District. Information for individuals who visited designated fever clinics, suspected COVID-19 cases, and confirmed cases were extracted through medical records at the aforementioned 5 hospitals, from January 16 to February 27, 2020. Field epidemiological reports were obtained from Changning District Center for Disease Control and Prevention to extract epidemiological and laboratory data of confirmed cases and their close contacts. Mann-Whitney U test was applied to identify the difference on time from symptom onset to COVID-19 diagnosis between outpatient monitoring and active case-finding in IBM SPSS 22.0 (IBM Corp., Armonk, NY).

A total of 22 local COVID-19 cases and 19 imported cases were detected in Changning District up to July 14,2020 . Of the 22 local cases, 8 (36.4\%) were detected by quarantining the people with Hubei Province travel history, $8(36.4 \%)$ were found by outpatient monitoring at designated fever clinics, and 6 $(27.3 \%)$ were identified by contact tracing (Figure 2). Of the 11 cases with travel history to Hubei Province,
4 arrived in Shanghai before the implementation of quarantine and were identified by outpatient monitoring. For those with close contacts with confirmed cases, 2 had suspected symptoms before their index cases confirmed with COVID-19, thus were found through other approaches rather than contact tracing.

From onset of symptoms to COVID-19 diagnosis, the median time for cases detected from outpatient monitoring, exposed people quarantining, and contact tracing was 6 days [Interquartile Range(IQR): 4-8], 2.5 days (IQR: 2-3) and 2 days (IQR: 1.5-3), respectively. Compared to outpatient monitoring, active case finding approaches significantly reduced the time from symptom onset to diagnosis $(P=0.003)$.

Local COVID-19 cases detected by outpatient monitoring were interspersed between January 27 and February 9, 2020. The total number of outpatient visits to designated fever clinics was 6,160 from January 16 to February 27, and 58 were identified as suspected cases. As a result, $8(13.8 \%)$ patients were confirmed with COVID-19, including one asymptomatic infection. The median duration from their first outpatient visit to diagnosis was 2.5 days (range, 0-10.0).

A total of 2,591 individuals reported themselves having a travel history to the Hubei Province over the past 14 days. Of them, $1,570 \quad(60.6 \%)$ were quarantined in Changning District while the rest were quarantined in other districts of Shanghai. During the 14-day quarantine, $0.6 \%$ of them $(9 / 1,570)$ reported fever, cough, diarrhea, or other symptoms, and 8 were confirmed as COVID-19 cases.

Overall, $6(22.2 \%)$ cases were detected from the 27 close contacts who resided in Changning District, including one confirmed after 2 reverse-transcription polymerase chain reaction tests. During the 14-day quarantine, $6(22.2 \%)$ of them reported fever and 3 were confirmed as COVID-19 cases. Another $2(7.4 \%)$ close contacts reported diarrhea or other respiratory symptoms without fever and were subsequently confirmed as COVID-19 cases. One asymptomatic infection was confirmed in a 1-year-old child, who was the youngest COVID-19 case in Changning District. (Figure 3)

\section{DISCUSSION}

This study delineated the COVID-19 case finding approaches at the earliest outbreak stage in Changning District with regard to key time points and 


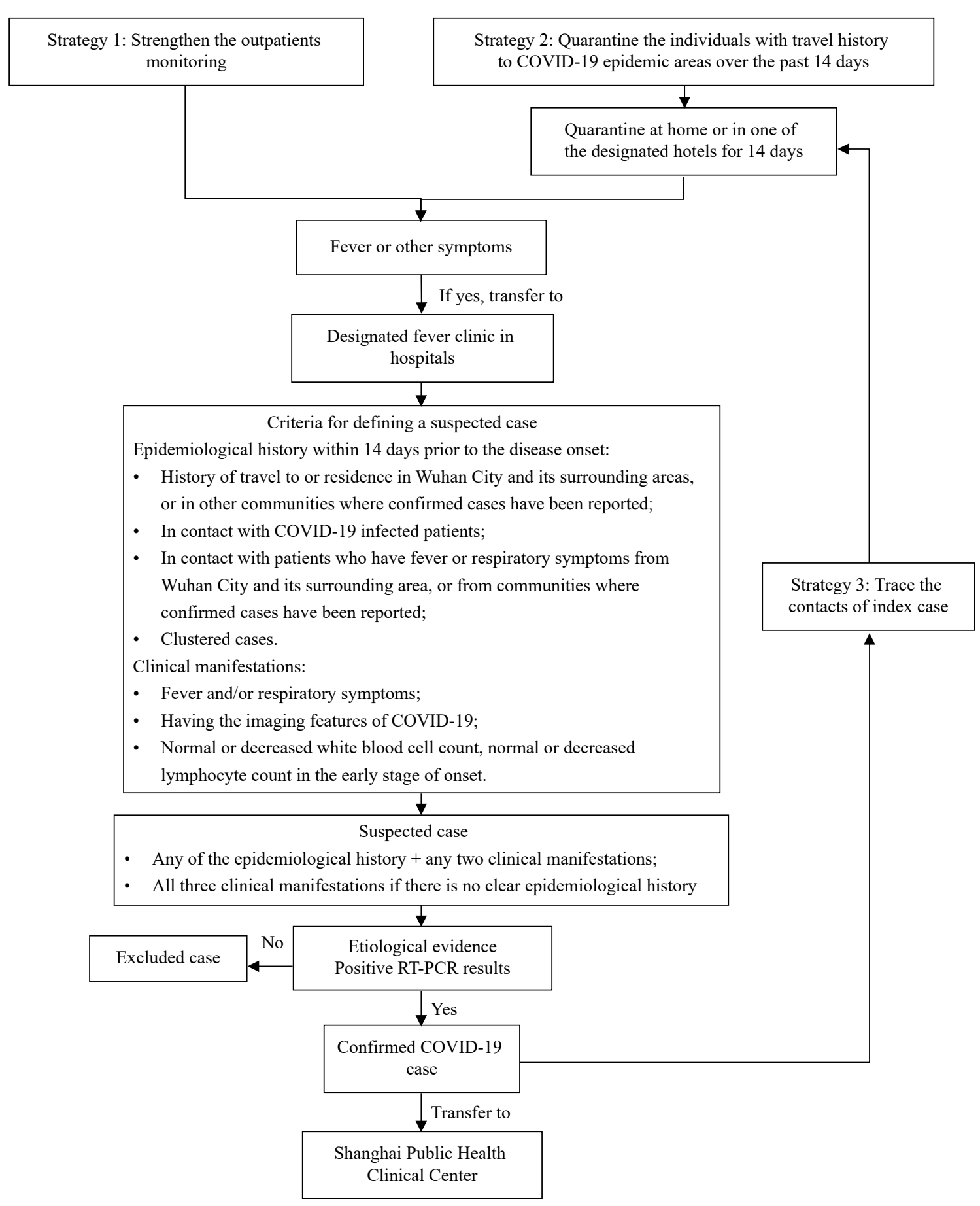

FIGURE 1. Flowchart for case finding approaches and case management of coronavirus disease 2019 (COVID-19) in Changning District, Shanghai Municipality.

Abbreviation: RT-PCR=reverse-transcription polymerase chain reaction.

epidemiological links. Compared to outpatient monitoring at designated fever clinics, active casefinding approaches, including quarantine of people with travel history to/from high-risk locations and contact tracing, significantly shortened the time from symptom onset to COVID-19 diagnosis, thereby reducing the risk of disease transmission in the population.

Experiences from the severe acute respiratory syndrome (SARS) epidemic suggested that the primary mode of SARS transmission was close contact with a symptomatic patient (5-6). As the pathogens for SARS and COVID-19 belong to the same coronaviridae family, it is reasonable to consider the close contacts of 


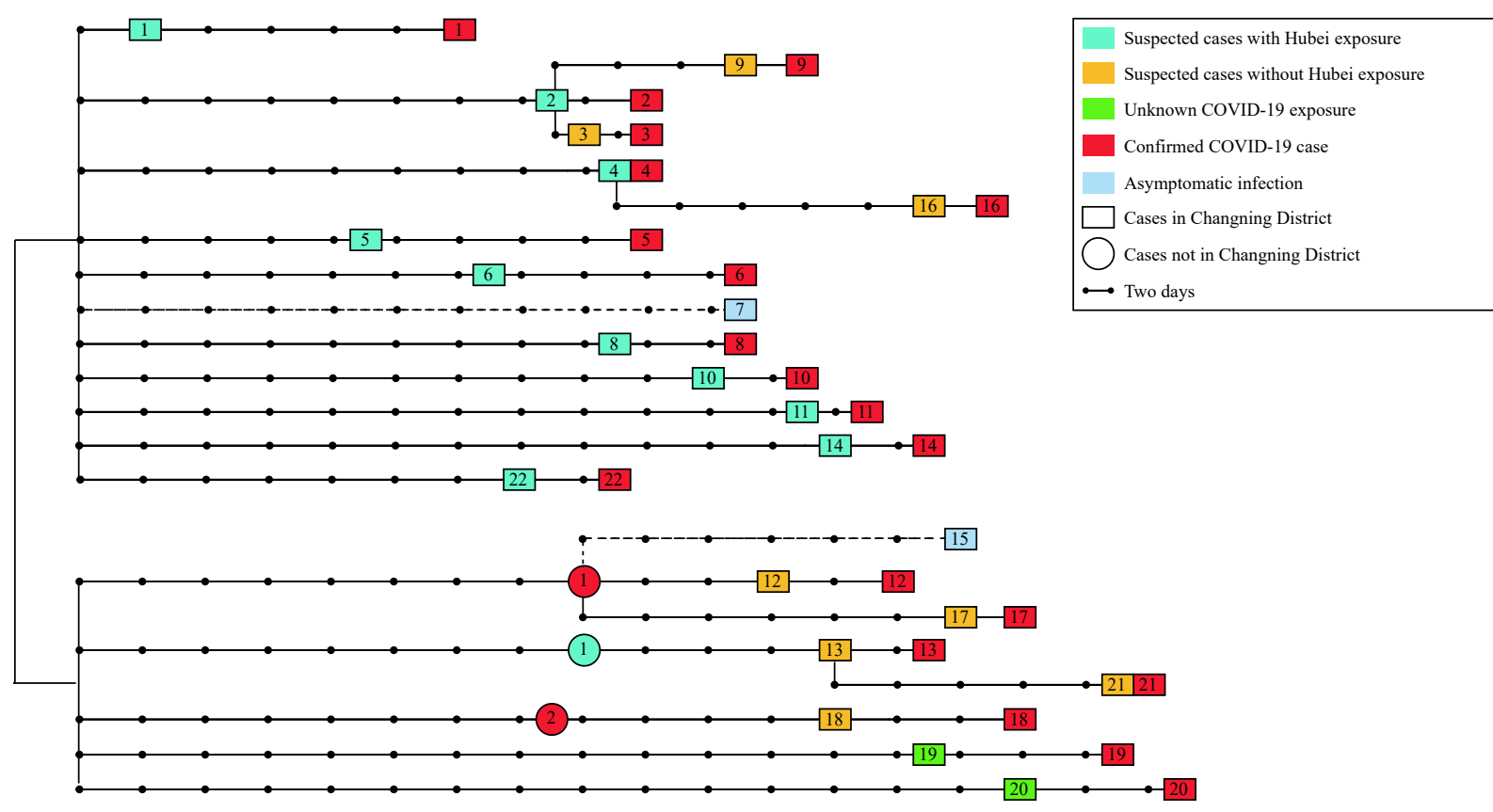

FIGURE 2. Epidemiological links of coronavirus disease 2019 (COVID-19) in Changning District, Shanghai Municipality.

\begin{tabular}{|c|c|c|}
\hline COVID-19 cases & Date & Close contacts \\
\hline \multirow[t]{3}{*}{ Patient 22} & $2020 / 1 / 25$ & $\mathrm{P} 22-\mathrm{C} 1$ \\
\hline & & $\mathrm{P} 22-\mathrm{C} 2$ \\
\hline & & P22-C3 \\
\hline \multirow[t]{3}{*}{ Patient 2\&3 } & $2020 / 1 / 26$ & $\mathrm{P} 2 \& 3-\mathrm{C} 1$ \\
\hline & & $\mathrm{P} 2 \& 3-\mathrm{C} 2$ \\
\hline & & P2\&3-C3 \\
\hline \multirow[t]{4}{*}{ Patient 4} & $2020 / 1 / 26$ & $\mathrm{P} 4-\mathrm{C} 1$ \\
\hline & & $\mathrm{P} 4-\mathrm{C} 2$ \\
\hline & & $\mathrm{P} 4-\mathrm{C} 3$ \\
\hline & & $\mathrm{P} 4-\mathrm{C} 4$ \\
\hline \multirow[t]{4}{*}{ Patient 5} & $2020 / 1 / 26$ & $\mathrm{P} 5-\mathrm{Cl}$ \\
\hline & & $\mathrm{P} 5-\mathrm{C} 2$ \\
\hline & & $\mathrm{P} 5-\mathrm{C} 3$ \\
\hline & & $\mathrm{P} 5-\mathrm{C} 4$ \\
\hline Patient 10 & $2020 / 1 / 31$ & $\mathrm{P} 10-\mathrm{C} 1$ \\
\hline \multirow[t]{4}{*}{ Patient 8} & $2020 / 1 / 29$ & $\mathrm{P} 8-\mathrm{C} 1$ \\
\hline & & $\mathrm{P} 8-\mathrm{C} 2$ \\
\hline & & $\mathrm{P} 8-\mathrm{C} 3$ \\
\hline & & P8-C4 \\
\hline \multirow[t]{5}{*}{ Patient X } & Unknown & PX-C1 \\
\hline & & $\mathrm{PX}-\mathrm{C} 2$ \\
\hline & & PX-C3 \\
\hline & & PX-C4 \\
\hline & & PX-C5 \\
\hline Patient 11 & $2020 / 2 / 2$ & $\mathrm{P} 11-\mathrm{C} 1$ \\
\hline Patient 13 & $2020 / 2 / 4$ & $\mathrm{P} 13-\mathrm{C} 1$ \\
\hline Patient 20 & $2020 / 2 / 12$ & $\mathrm{P} 20-\mathrm{C} 1$ \\
\hline
\end{tabular}

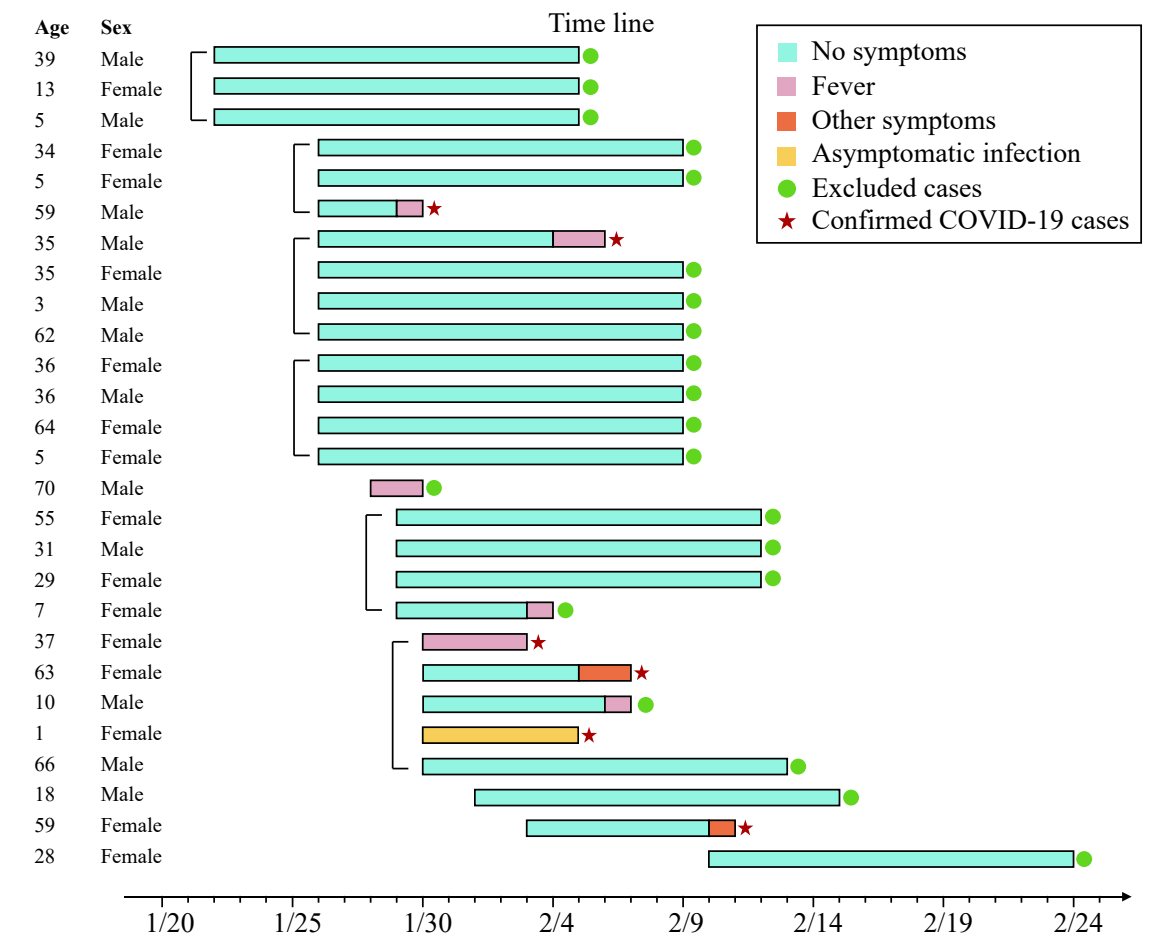

FIGURE 3. Contact tracing for confirmed coronavirus disease 2019 (COVID-19) cases in Changning District, Shanghai Municipality.

Note: Date of COVID-19 diagnosis was shown for each index cases. Patient $X$ was detected in another district of Shanghai and thus lacked a COVID-19 diagnosis date. Excluded cases included those who completed 14-day quarantine without any symptoms and those who had suspected symptoms during quarantine but received negative reverse-transcription polymerase chain reaction results twice.

COVID-19 cases as a high-risk population for quarantine, especially with confirmed evidence on human-to-human transmission (7-8). In this study, $20.0 \%$ of close contacts were subsequently confirmed 
with COVID-19, supporting the high risk of infection in this population. It would be ideal to provide pathogenic testing for all identified close contacts, especially at the early stage of COVID-19 outbreak.

In this study, outpatient monitoring at designated fever clinics and quarantining people who had travelled to COVID-19 epidemic areas also played an important role in disease containment. Despite the requirement of a substantial amount of time and labor to implement quarantine, this approach effectively detected nearly one-third of COVID-19 cases. Another one-third of cases were detected by outpatient monitoring, although it was associated with a longer time from symptom onset to COVID-19 diagnosis. The unique strength of outpatient monitoring is its ability to identify early cases before quarantining exposed people and contact tracing are implemented. In addition, outpatient monitoring can provide additional assurance by catching patients who might have been missed by the active case-finding approaches.

This study was subject to some limitations. First, this study only had exposure and diagnosis information, and therefore, was unable to prove the epidemiological links between COVID-19 cases at the genomic level. Second, due to the presence of mild symptoms and asymptomatic infection, some COVID-19 cases might have been missed, resulting in under-ascertainment. However, since the last case was reported on February 12, there had been no new domestic cases detected since then, indicating that the case-finding approaches implemented in Changning District were probably effective to achieve disease containment.

In conclusion, contact tracing of confirmed COVID-19 cases was effective to find new cases. Compared to outpatient monitoring at designated fever clinics, active case-finding approaches significantly reduced the time from symptom onset to COVID-19 diagnosis, therefore reducing the risk of disease transmission. The case-finding approaches implemented in Changning District was effective in rapid COVID-19 containment and may help inform public health policy in other regions where strict lockdown is not applicable.

Conflicts of interest: No conflicts of interest.

doi: $10.46234 / \mathrm{ccdcw} 2021.149$

\# Corresponding author: Biao Xu, bxu@shmu.edu.cn.

1 Department of Epidemiology, School of Public Health, Fudan University, Shanghai, China; ${ }^{2}$ Key Lab of Health Technology Assessment, National Health Commission of the People's Republic of China, Fudan University, Shanghai, China; ${ }^{3}$ Changning District Center for Disease Control and Prevention, Shanghai, China.

${ }^{\&}$ Joint first authors.

Submitted: May 06, 2021; Accepted: May 19, 2021

\section{REFERENCES}

1. Jones BA, Betson M, Pfeiffer DU. Eco-social processes influencing infectious disease emergence and spread. Parasitology 2017;144(1):26 36. http://dx.doi.org/10.1017/S0031182016001414

2. Shanghai traffic command center. Analysis of ten years data of the Hongqiao integrated transportation Hub. Shanghai, China. 2019. http:// news.carnoc.com/list/511/511777.html. [2020-3-26]. (In Chinese).

3. Hellewell J, Abbott S, Gimma A, Bosse NI, Jarvis CI, Russell TW, et al. Feasibility of controlling COVID-19 outbreaks by isolation of cases and contacts. Lancet Glob Health 2020;8(4):e488 - 96. http://dx.doi.org/ 10.1016/S2214-109X(20)30074-7.

4. Aleta A, Martín-Corral D, Piontti APY, Ajelli M, Litvinova M, Chinazzi $\mathrm{M}$, et al. Modelling the impact of testing, contact tracing and household quarantine on second waves of COVID-19. Nat Hum Behav 2020;4(9):964 - 71. http://dx.doi.org/10.1038/s41562-020-0931-9.

5. Booth CM, Matukas LM, Tomlinson GA, Rachlis AR, Rose DB, Dwosh HA, et al. Clinical features and short-term outcomes of 144 patients with SARS in the greater Toronto area. JAMA 2003;289(21):2801 - 9. http://dx.doi.org/10.1001/jama.289.21.JOC30885.

6. Hsu LY, Lee CC, Green JA, Ang B, Paton NI, Lee L, et al. Severe acute respiratory syndrome (SARS) in Singapore: clinical features of index patient and initial contacts. Emerg Infect Dis 2003;9(6):713-7. http://dx.doi.org/10.3201/eid0906.030264.

7. Li Q, Guan XH, Wu P, Wang XY, Zhou L, Tong YQ, et al. Early transmission dynamics in Wuhan, China, of novel coronavirus-infected pneumonia. N Engl J Med 2020;382(13):1199 - 207. http://dx.doi.org/ 10.1056/NEJMoa2001316.

8. Chan JFW, Yuan SF, Kok KH, To KKW, Chu H, Yang J, et al. A familial cluster of pneumonia associated with the 2019 novel coronavirus indicating person-to-person transmission: a study of a family cluster. Lancet 2020;395(10223):514 - 23. http://dx.doi.org/10.1016/S01406736(20)30154-9. 